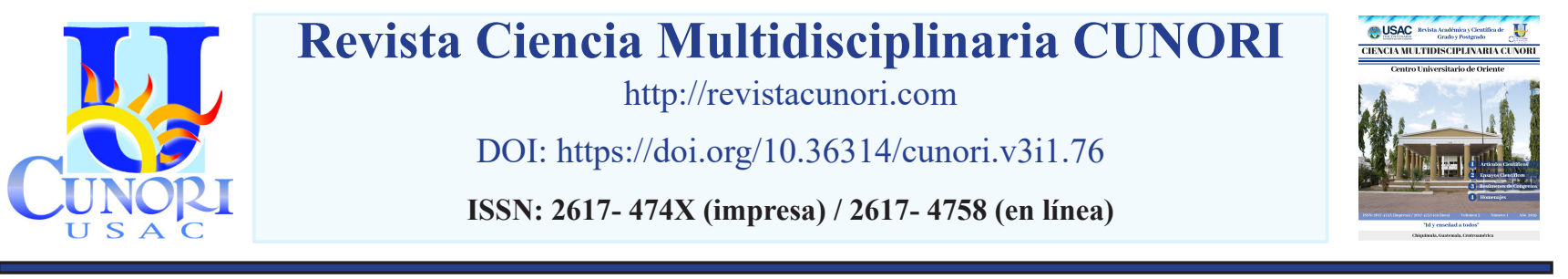

Como citar el artículo

Aguilar, G., Mazariegos, E., Arriola, C., Peralta, S., (2019). Caracterización de pacientes con hijos diagnosticados con malformaciones congénitas. Revista Ciencia Multidisciplinaria CUNORI, 3(1), 19-28. DOI: https://doi.org/10.36314/cunori.v3i1.76

\title{
Caracterización de pacientes con hijos diagnosticados con malformaciones congénitas
}

\section{Characterization of patients with children diagnosed with congenital malformations}

\author{
Glida Aguilar*, Edvin Mazariegos, Carlos Arriola, Sara Peralta \\ Centro Universitario de Oriente (CUNORI), Universidad de San Carlos de Guatemala
}

Recibido: 21 de julio de 2018 / Revisión: 20 de agosto de 2018 / Aceptado: 10 de enero de 2019

Disponible en internet el 30 de agosto de 2019

Resumen

*Autor para correspondencia.

Correo electrónico: glidaross1391@gmail.com

$\mathrm{L}$ as malformaciones congénitas son patologías que se producen en etapas embrionario-fetales, pudiendo ser defectos estructurales o funcionales, teniendo un sin número de posibles causas que abarcan desde genéticas, físicas, o incluso ambiental. Es un estudio descriptivo retrospectivo el cual caracterizó a 230 pacientes con hijos diagnosticados con malformaciones congénitas ingresadas en el Hospital Nacional de Chiquimula. Se logró comprobar que el grupo etario predominante fueron las comprendidas entre 23 a 32 años con un 43\% (98) de los casos. La ocupación predominante fue la de ama de casa con un 97\% (224), en cuanto a la escolaridad, el $60 \%$ era analfabeta (138). El 100\% no poseía antecedentes de malformaciones congénitas (230). El 5\% presentaba hipertensión arterial crónica (12), el 1\% presentaba diabetes mellitus II, síndrome asmático y síndrome convulsivo (3). El 7\% afirmó haber consumido medicamentos durante el embarazo, (IECAS, Metformina, Ácido Valproico, y Salbutamol) (15). El 70\% afirmó haber llevado un adecuado control prenatal (160), el 97\% no se realizó ningún ultrasonido obstétrico (223), el 62\% afirmó consumir suplementación vitamínica durante el embarazo haciendo la consideración que la mayor parte las consumió durante el último trimestre (143). El 77\% afirmó no haber tenido abortos (177). La malformación congénita predominante fue hidrocefalia con un $16 \%$ (40) seguido de mielomeningocele correspondiendo a un 14\% (36).

Palabras clave: malformación congénita, caracterización, hijos, diagnóstico

\section{Abstract}

The congenital defects are pathologies occur in stages embryonic-fetal may be defects structural and functional, having a countless possible causes ranging from genetic, physical, or even environmental. Retrospective descriptive study that characterized 230 patients with children diagnosed with congenital malformations in National Hospital of Chiquimula. We were able to prove the age group predominant are the fall between 23 to 32 whit 43\% (98) of the cases, occupation predominant was that of housewife with $97 \%$ (224), in terms of schooling, 60\% was illiterate (138), the 100\% no had a history of congenital malformations (230), the $5 \%$ had high blood pressure chronic (12), 1\% has diabetes mellitus II, syndrome asthma and syndrome seizure (3), the 7\% said to have used drugs during pregnancy, (IECAS, Metformin, acid valproic and salbutamol) (15), the 70\% said to have taken a suitable prenatal care (160), the $97 \%$ was not any ultrasound obstetrical (223), the 62\% said consume supplementation vitamin during pregnancy doing consideration that most of the consumed during the last quarter (143). The 77\% said not have had abortion (177). The malformation congenital predominant was hydrocephalus with $16 \%$ (40) followed by myelomeningocele corresponding to $14 \%(36)$.

Keywords: congenital, defects, characterization, children, diagnosis 


\section{Introducción}

Las malformaciones congénitas son patologías que se producen en etapas embrionario-fetales, pudiendo ser defectos estructurales o funcionales, de los cuales se pueden detectar por medio de la inspección física o con estudios especializados, teniendo un sin número de posibles causas que abarcan desde genéticas, físicas, o incluso ambiental. Las malformaciones congénitas se clasifican en anomalías mayores o menores diferenciándolas según puedan limitar significativamente la función normal de la parte afectada o reduciendo la expectativa de vida del paciente. La organización mundial de la salud estima que alrededor de 276,000 recién nacidos fallecen durante las primeras cuatro semanas de vida debido a malformaciones congénitas

La etiología de las malformaciones congénitas en la mayoría de los casos es incierta, pero se sabe que el bajo nivel socioeconómico puede ser un determinante indirecto para esta problemática, identificándose a nivel mundial cerca de1 $94 \%$ de las malformaciones congénitas graves o mayores se producen en países en vías de desarrollo, en los que la mayoría de las mujeres en edad reproductiva carecen de una adecuada alimentación y exposición a agentes químicos como plaguicidas, tabaco, alcohol o radiación que producen o aumentan la incidencia de alteraciones en el desarrollo prenatal (OMS 2015).

En el presente estudio se realizó la caracterización de las pacientes con hijos diagnosticados con malformaciones congénitas ingresadas en el Hospital Nacional de Chiquimula durante enero 2013 a diciembre 2017 mediante la revisión de 230 expedientes clínicos, se determinó que el rango de edad mayormente afectado fueron las comprendidas entre los 23 a 32 años, la mayoría analfabetas, con un adecuado control prenatal y suplementación vitamínica, las cuales la mayor parte no tenían ultrasonido obstétrico y con un pequeño porcentaje de pacientes expuestas a teratógenos. Por otra parte, se determinó que no había un registro clínico completo y adecuado que permita realizar actividades para disminuir y prevenir esta patología.

\section{Materiales y métodos}

La investigación realizada es de carácter descriptivo retrospectivo siendo el objetivo de este tipo de estudio la observación de características de interés en un lapso de tiempo, siendo estas características: la edad de la paciente, procedencia, ocupación, estado civil, escolaridad, religión, numero de gestas, número de partos, número de cesáreas, número de abortos, control prenatal, lugar del control prenatal, utilización de ultrasonido obstétrico, suplementación durante el embarazo, antecedentes médicos, antecedentes quirúrgicos, antecedentes toxicológicos, y el tipo de malformación congénita encontrada. Para dar respuesta a las preguntas generadoras y, por ende; cumplir con los objetivos planteados, se utilizó el método cualitativo que se caracteriza por permitir un mayor acercamiento con el sujeto de estudio, siendo estas las pacientes con hijos diagnosticados con malformaciones congénitas.

Con este tipo de método, se buscó por medio de una boleta de recolección de datos, la revisión de papeletas de interés que poseían el diagnostico de pacientes con hijos diagnosticados con malformaciones congénitas. Cabe mencionar que la muestra no se estableció cuantitativamente o mediante un procedimiento estadístico, en cambio se tomaron en cuenta el acceso a las papeletas con la información requerida. 
El universo estuvo constituido por doscientas treinta papeletas, comprendidas entre los años enero 2013 a diciembre 2017. En cuanto a los criterios de selección, se tomó en consideración el muestreo intencional, siendo el referente las pacientes con hijos diagnosticados con malformaciones congénitas durante el periodo de enero 2013 a diciembre 2017.

I

Además, se debe mencionar que el procesamiento y análisis de la información recolectada se realizó de la siguiente manera: se distribuyó los datos de forma manual según los períodos de tiempo en que fueron realizados en el estudio, se procesó los datos en Microsoft Excel según las variables incluidas en la operacionalización de las variables, se tabuló los datos en frecuencias simples para realizar el análisis de la información de cada variable y se realizó las gráficas para expresar cada variable. La boleta estuvo constituida por cuatro series, la primera serie se denominó "Datos Generales" en el cual se tomó en cuenta la edad, profesión u oficio, procedencia y escolaridad. La segunda serie se denominó "Datos Obstétricos" en la que se incluyeron datos obstétricos de interés como por ejemplo gestas, partos, abortos, cesáreas, exposición a rayos X, malformaciones congénitas anteriores, control prenatal, el lugar del control prenatal, ultrasonidos controles y la cantidad de estos, suplementación vitamínica y se detalló las posibilidades de inmunizaciones.

La tercera serie se denominó “Antecedentes Maternos” en el cual se incluyeron todos los datos de interés maternos, lo que se pueden mencionar: Antecedentes médicos, quirúrgicos, traumáticos, toxicológicos y alérgicos. La cuarta y última serie se dominó "Malformación Congénita" en la cual se enlistó según la clasificación de malformaciones congénitas los diferentes tipos de anomalías congénitas más comunes y se contó con una opción de "otros defectos congénitos" si la anomalía congénita encontrada no se tomó en cuenta en el listado de las más comunes. Se define como malformación congénita a las anormalidades estructurales o funcionales, que generalmente ocurren durante el desarrollo del feto, y que es posible detectarlo durante el embarazo, el parto o durante el desarrollo temprano. En muchos países las malformaciones congénitas son una de las causas importantes de discapacidad, mortalidad infantil y enfermedad crónica. Causando gran impacto en el afectado, la familia, sistemas de salud y sociedad en general (Ortiz, 2003).

Existe diferentes clasificaciones para las malformaciones congénitas que se puedan presentar, pero en general se clasifican en mayores aquellas alteraciones que limitan de alguna manera al individuo y que requieren asistencia altamente especializada para una adecuada y correcta integración al medio; y menores siendo estas alteraciones que limitan muy poco al individuo y que no incurren en asistencia especializada; teniendo en este tipo de alteraciones problemas mayormente estéticos que funcionales (Vega, 2005).

La organización mundial de la salud estima que alrededor de 276,000 recién nacidos fallecen durante las primeras cuatro semanas de vida debido a malformaciones congénitas, pudiendo estas tener un origen genético, infeccioso, o ambiental, aunque en la mayoría resulta muy difícil identificar la principal causa de este trastorno.

No obstante, el bajo nivel socioeconómico puede ser un determinante indirecto para esta problemática identificándose a nivel mundial cerca del 94\% de las malformaciones congénitas graves o mayores se producen en países en vías de desarrollo, en los que la mayoría de las mujeres en edad reproductiva ca- 
recen de una adecuada alimentación y exposición a agentes químicos como plaguicidas, tabaco, alcohol o radiación que producen o aumentan la incidencia de alteraciones en el desarrollo prenatal.

Por otra parte, los extremos de la vida también aumentan significativamente la probabilidad de tener malformaciones congénitas, siendo el síndrome de Down la principal alteración cromosómica en madres añosas. Entre las causas genéticas; la consanguinidad observada mayormente en comunidades étnicas como judíos o finlandeses, aumenta significativamente la prevalencia de malformaciones congénitas genéticas raras y multiplica el riesgo de muerte neonatal e infantil. El poco control prenatal, al igual que infecciones maternas graves como la sífilis, rubeola entre otras son causa importante de esta patología (OMS 2015). Lo que puede orientar a los diferentes factores de riesgo para la población chiquimulteca, especialmente a mujeres en edad fértil y embarazadas para el desarrollo de malformaciones congénitas, entre los cuales destacan; edad de la paciente embarazada, ya que se sabe que los extremos de este están mayormente asociados al desarrollo de anormalidades congénitas tales como es el caso de mujeres añosas para el desarrollo de productos con síndrome de Down.

Factores de riesgo como ocupación están asociados también a esta patología ya que la población se dedica en su mayoría a la agricultura teniendo como riesgo la exposición prolongada a pesticidas o agentes teratógenos.

Por otro parte la consanguinidad aumenta considerablemente la tasa de tener malformaciones congénitas, es bien sabido que la falta de educación y múltiples problemas sociales lo hacen una práctica común, asociado a esto la falta de control prenatal y el consumo de suplementaciones vitamínicas acrecienta estas patologías. Teniendo en cuenta todas estas variables y otras de suma importancia es importante enfatizar en estudios que caractericen a las mujeres con hijos diagnosticados con malformaciones congénitas ya que el consenso de todos estos factores de riesgo son determinantes en esta patología, por lo anterior se hace de gran interés y con alto impacto poblacional la realización de dicho estudio y con ello lograr identificar a dicha población catalogándola de alto riesgo y con ello disminuir la incidencia de malformaciones congénitas.

En los últimos años, numerosos estudios han examinado los posibles efectos teratogénicos del consumo de tabaco durante el embarazo. El cigarrillo, específicamente, contiene al menos 55 elementos carcinogénicos. Cabe destacar que, aunque existe controversia en cuanto a si fumar durante el embarazo puede causar malformaciones congénitas específicas, se han estudiado especialmente los efectos en el pulmón y la presencia de hendiduras orofaciales (Cisneros, 2014). 


\section{Resultados}

El grupo etario predominante en el presente estudio, fueron las pacientes comprendidas entre 23 a 32 años con un $43 \%$ correspondiente a 98 casos, el cual se determinó que el 59\% de estas eran de los diferentes municipios del departamento de Chiquimula, 97\% eran amas de casa, 53\% se encontraban unidas, $42 \%$ eran pertenecientes a la religión evangélica y el $60 \%$ es población analfabeta.

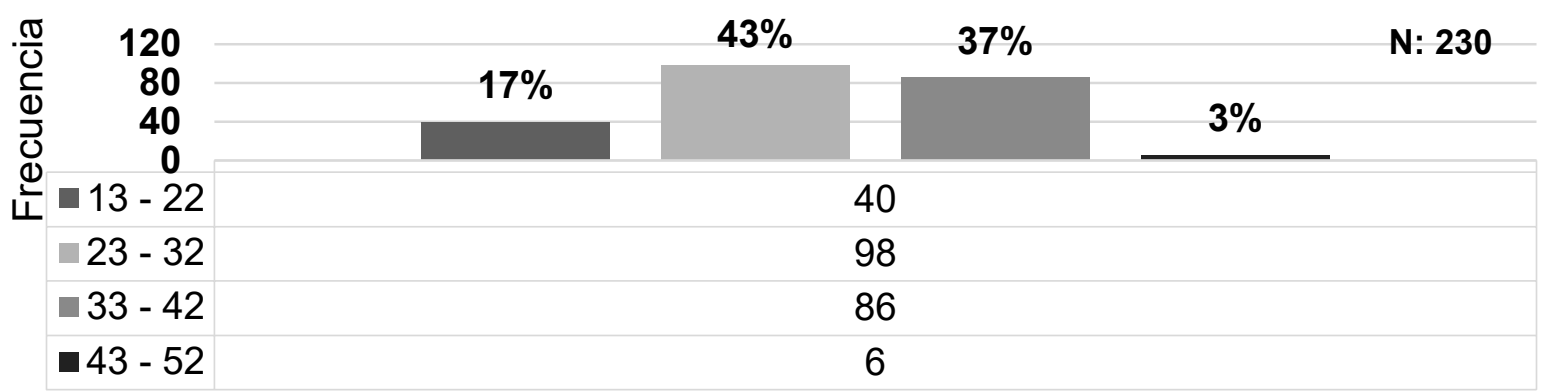

Figura 1: Distribución según el rango de edad de las pacientes con hijos diagnosticados con malformaciones congénitas, ingresados en el Hospital Nacional de Chiquimula durante el período de enero de 2013 a diciembre de 2017.

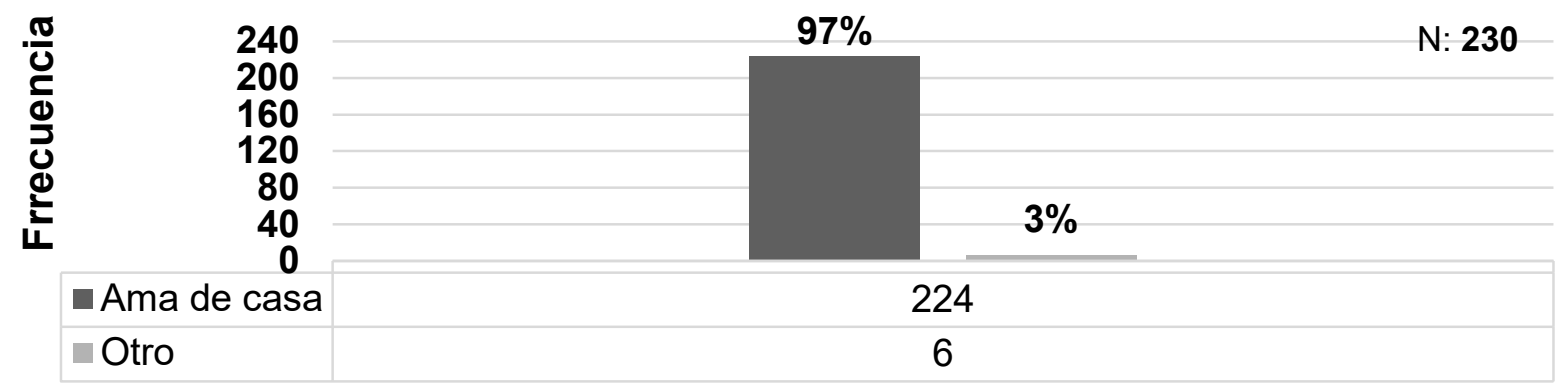

Figura 2: Distribución según la ocupación de las pacientes con hijos diagnosticados con malformaciones congénitas, ingresados en el Hospital Nacional de Chiquimula durante el período de enero de 2013 a diciembre de 2017.

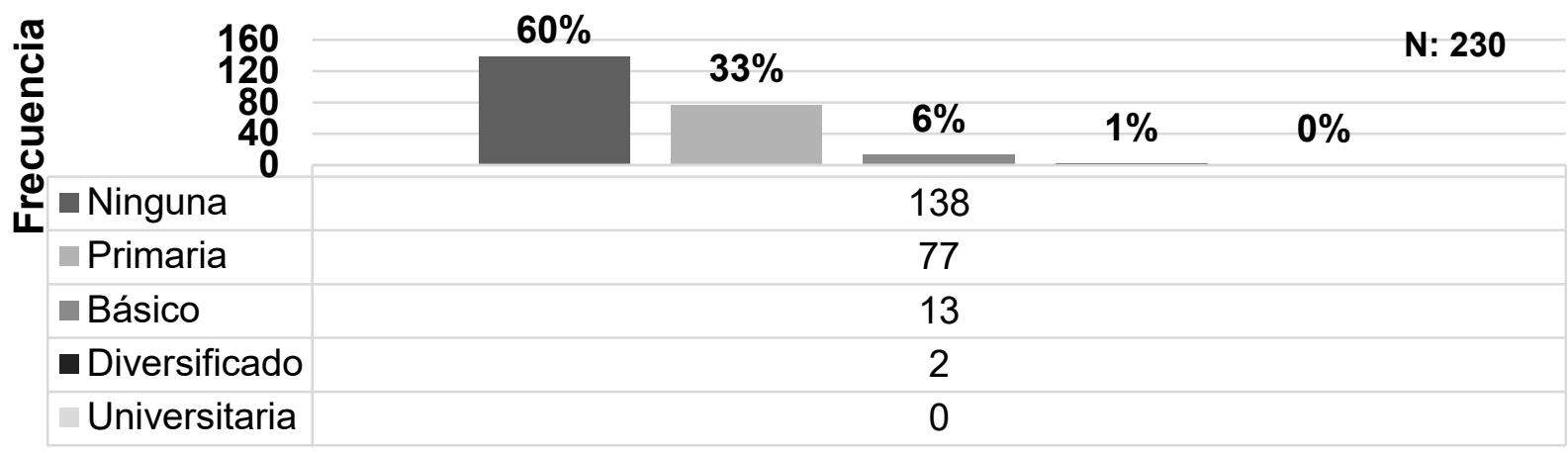

Figura 3: Distribución según la escolaridad de las pacientes con hijos diagnosticados con malformaciones congénitas, ingresados en el Hospital Nacional de Chiquimula durante el período de enero de 2013 a diciembre de 2017. 
Dentro de los antecedentes gineco-obstétricos, el 47\% se encontraban en su primera o segunda gesta, 44\% habían tenido uno o ningún parto, $66 \%$ no habían tenido cesáreas, $77 \%$ no habían tenido ningún aborto, y el 100\% no tenía antecedentes de malformaciones congénitas.

En cuanto a los antecedentes médicos, el 94\% afirmó no poseer ningún antecedente de importancia, el $5 \%$ presentaba hipertensión arterial crónica y el 1\% otros antecedentes médicos como diabetes mellitus II, síndrome asmático y síndrome convulsivo. El 80\% no presentaron antecedentes quirúrgicos, mientras que el $20 \%$ poseían una cesárea anterior. Por otra parte, el $100 \%$ de las pacientes no tenía ningún antecedentes traumáticos o alérgicos.

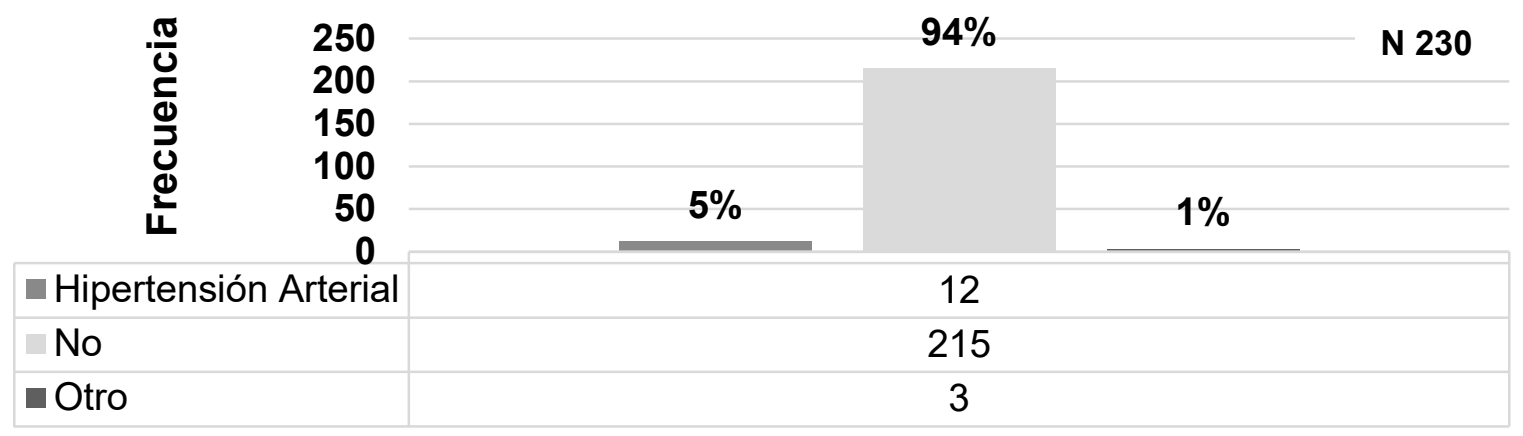

Figura 4: Distribución sobre antecedentes médicos las pacientes con hijos diagnosticados con malformaciones congénitas, ingresados en el Hospital Nacional de Chiquimula durante el período de enero de 2013 a diciembre de 2017.

Respecto a los antecedentes toxicológicos, el 7\% afirmó haber consumido algún tipo de medicamentos durante el embarazo, entre los que cabe mencionar los inhibidores de la enzima convertidora de angiotensinógeno, metformina, ácido valproico y salbutamol.

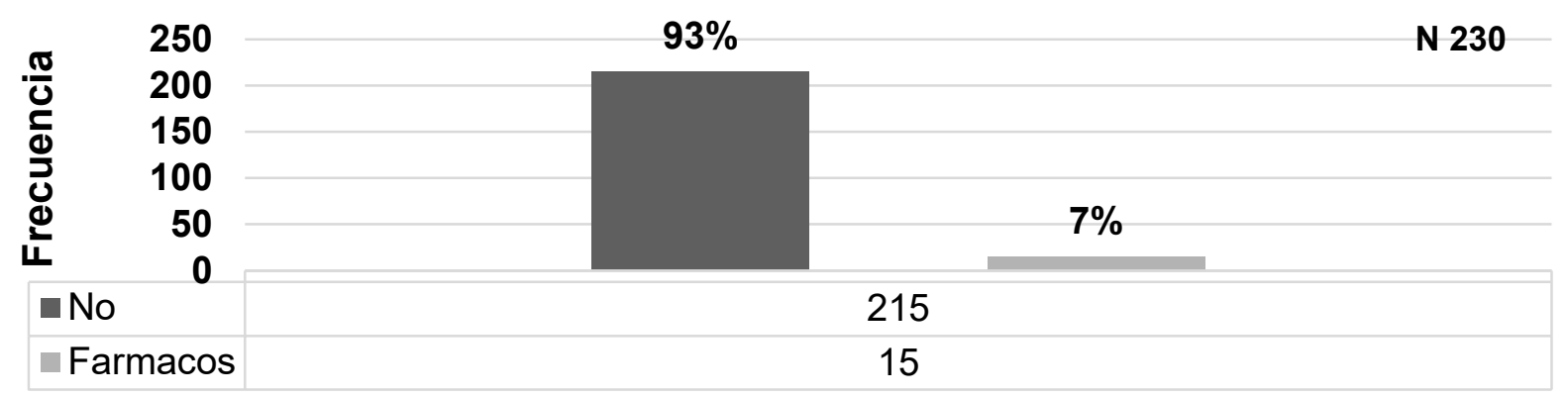

Figura 5: Distribución sobre antecedentes toxicológicos de las pacientes con hijos diagnosticados con malformaciones congénitas, ingresados en el Hospital Nacional de Chiquimula durante el período de enero de 2013 a diciembre de 2017. 
Acerca del control prenatal el 70\% de las pacientes afirmó si llevar un adecuado control prenatal, siendo este realizado en un $62 \%$ de estos en los puestos de salud, por otra parte, el $97 \%$ de las pacientes refirió no haberse realizado ningún ultrasonido obstétrico y el $62 \%$ haber tenido suplementación vitamínica durante el embarazo, haciendo la consideración que la mayor parte de estas pacientes se suplementó durante el último trimestre del embarazo, haciendo de esta manera infectiva la acción de estas contra malformaciones congénitas.

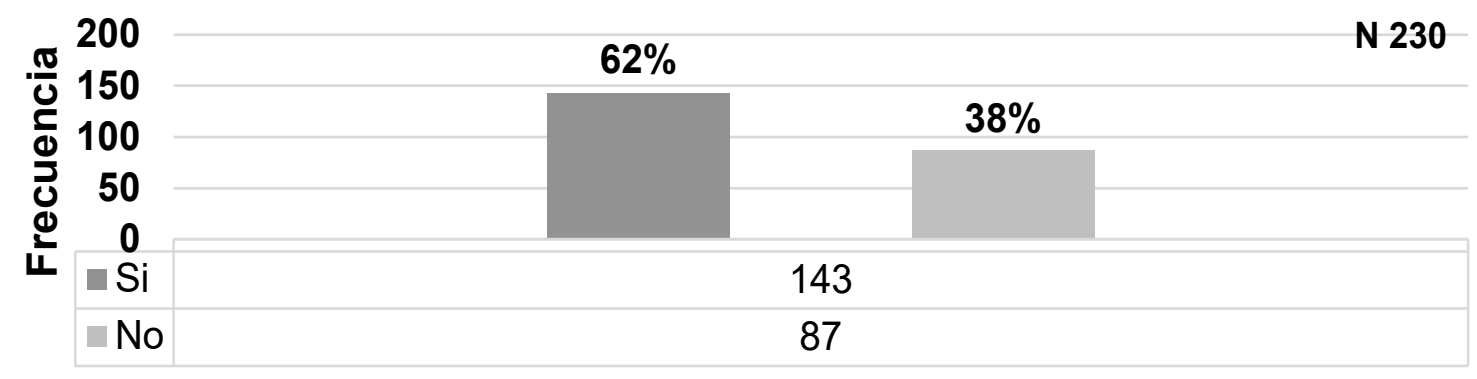

Figura 6: Distribución sobre la utilización de suplementación durante el embarazo de las pacientes con hijos diagnosticados con malformaciones congénitas, ingresados en el Hospital Nacional de Chiquimula durante el período de enero de 2013 a diciembre de 2017.

El 100\% de las pacientes estudiadas afirmo no tener ninguna enfermedad de transmisión sexual, no haber sido expuesta a rayos x y no haberse realizado ninguna inmunización durante el embarazo.

En cuanto a la clasificación de las malformaciones congénitas, se observó que el 21\% de estas anomalías estaba compuesta por diferentes diagnósticos entre los que se puede mencionar hidranencefalia, gastrosquisis, onfalocele, anencefalia entre otros, mientras que el 16\% correspondía a hidrocefalia, el 14\% a mielomeningocele, $11 \%$ a cardiopatías congénitas, $8 \%$ a pie equino varo, $7 \%$ a labio hendido, $6 \%$ a síndrome de Down y 5\% a paladar hendido, haciendo consideración que existieron varios diagnósticos en un mismo pacientes.

\section{Discusión}

Habiendo culminado la investigación en 230 pacientes con hijos diagnosticados con malformaciones congénitas ingresados en el Hospital Nacional de Chiquimula durante el período de enero de 2013 a diciembre de 2017, se pueden analizar y discutir diversos aspectos.

El año con mayor número de casos de pacientes con hijos diagnosticados con malformaciones congénitas fue el año 2016 con un total de 72 pacientes correspondiendo a un 31\% del total de los casos, seguido por el 2017 con un total de 69 pacientes correspondiendo a un 30\% del total de casos, pudiendo observarse un aumento considerable de los casos reportados en años anteriores, esto posiblemente debido a un mayor porcentaje de embarazos adolescentes, un inadecuado control prenatal y una insuficiente suplementación vitamínica durante el primer trimestre del embarazo. 
Se pudo observar importante fenómeno en cuanto a la edad de los casos estudiados, pues, las pacientes con hijos más diagnosticados con malformaciones congénitas no se encontraban en los extremos de la vida como es esperado según la literatura, el cual considera que las pacientes añosas y pacientes jóvenes tienden a tener mayor porcentaje de hijos con estas anomalías, se observó que el mayor porcentaje de pacientes con hijos diagnosticados con malformaciones congénitas se encontró en el grupo etario de 23 a 32 años correspondiente a un 43\%, mientras que en el grupo etario de 13 a 22 años solo se encontró un total de 40 pacientes correspondiendo a un $17 \%$ del total de la población estudiada y el grupo etario de 43 a 52 años considerados para esta investigación como el grupo con mayor probabilidad de diagnósticos de malformaciones congénitas se encontraron un total de 6 pacientes correspondiendo a un $3 \%$.

Otro dato importante sobre las pacientes con hijos diagnosticados con malformaciones congénitas es el lugar de procedencia, pues es bien sabido según la literatura que estas anomalías se dan en mayor proporción en pacientes residentes del área rural debido a ser consideradas áreas con mayor problemas socioeconómicos, carentes de una adecuada alimentación y tener mayor exposición a agentes químicos como plaguicidas; pero en la presente investigación se observó que el mayor porcentaje de pacientes con hijos diagnosticados con malformaciones congénitas son residentes de los distintos municipios del departamento de Chiquimula, con un total de 135 pacientes correspondiendo a un 59\%, contrastando con Jocotán y Camotán con un total de 20 pacientes correspondiendo a un $13 \%$.

En cuanto a la ocupación de las pacientes con hijos diagnosticados con malformaciones congénitas, se observó que el mayor porcentaje son amas de casa con 224 pacientes correspondiendo a un 97\% contrastando con un 3\% de otras ocupaciones entre las que se pueden mencionar, niñeras, estudiantes y agricultoras. Por otro lado, el estado civil de las pacientes dentro de esta investigación refirió estar unidas en un 53\% correspondiendo a 121 pacientes, esto pudiendo ser en la mayoría de los casos algo cultural o por falta de recursos económicos, el 26\% refirieron estar casadas correspondiendo a 60 pacientes las cuales gran parte de estas se encontraba en la adultez, mientras que únicamente el $21 \%$ correspondiendo a 49 pacientes refirieron estar solteras, debido a estar comprendidas en el rango de la adolescencia o adulto joven.

Se determinó durante la investigación, la escolaridad de las pacientes con hijos diagnosticados con malformaciones congénitas, observándose que el 60\% correspondiente a 138 pacientes no tienen ningún grado de escolaridad, se puede inferir que este porcentaje optan por abandonar sus estudios en prioridad de contribuir económicamente a seno familiar, seguido por $33 \%$ correspondiente a 77 pacientes con escolaridad primaria y únicamente el $1 \%$ correspondiendo a 2 personas poseen el nivel diversificado de escolaridad.

Dentro de las gestas de las pacientes con hijos diagnosticados con malformaciones congénitas, la literatura indica que pacientes con múltiples gestas son propensas a tener hijos con este diagnóstico, sin embargo se determinó que el $47 \%$ correspondiente a 109 pacientes eran primigestas o secundigestas mientras que el $22 \%$ correspondientes a 52 pacientes fueron catalogadas como multíparas, por lo que se puede observar que no se correlaciona el resultado obtenido con la literatura; esto debido a que en la mayoría de los casos a pesar de ser pacientes que llevaron un adecuado control prenatal con un 70\% correspondiendo a 160 pacientes, estas fueron atendidas en un $62 \%$ correspondiendo a 99 pacientes en puestos de salud, en el cual la mayoría de los casos estos lugares no cuentan con personal calificado 
y equipo adecuado como ultrasonidos de primer nivel para determinar algún tipo de malformación congénita.

Por otra parte, la suplementación con ácido fólico y sulfato ferroso es la piedra angular para la prevención de malformaciones congénitas, teniendo en consideración que esta suplementación se debe iniciar con un periodo mínimo de tres meses antes de la concepción, durante la investigación se determinó que un $62 \%$ correspondientes a 143 pacientes afirmaron haber tomado suplementación y únicamente el $38 \%$ correspondiente a 87 pacientes refirieron no haber consumido ácido fólico y sulfato ferroso, observando que a pesar de haber consumido suplementos vitamínicos durante el embarazo, estas pacientes presentaron algún tipo de anomalía congénita debido a que en su mayoría consumieron los suplementos en el último trimestre del embarazo en el cual la acción preventiva de estos medicamente se pierde.

Respecto a los antecedentes médicos de las pacientes con hijos diagnosticados con malformaciones congénitas se determinó que el $94 \%$ correspondiente a 215 pacientes no refirieron antecedentes médicos de importancias, mientras que el 5\% correspondiente a 12 pacientes afirmaron padecer hipertensión arterial crónica; es bien sabido por la literatura que padecer enfermedades como hipertensión arterial crónica y diabetes mellitus durante el embarazo ocasiona un sin número de problemas tanto para el feto como para la paciente, por lo que llevar un adecuado control prenatal durante el embarazo es de suma importancia para el monitoreo de ambos; las 12 pacientes que afirmaron poseer hipertensión arterial crónica tenían como antecedente toxicológico el uso de fármacos inhibidores de la enzima convertidora de angiotensinógeno (IECA) durante el embarazo, teniendo en cuenta este dato, es conocido que los IECAS están catalogados tipo "C" durante el primer trimestre debido a tener altas tasas de muerte fetal y malformaciones congénitas y tipo " $\mathrm{D}$ " durante el segundo y tercer trimestre ya que provocan toxicidad fetal y neonatal, pudiendo ser estos medicamentos el causante de las malformaciones congénitas encontradas en estas pacientes.

Por ultimo en cuanto a la distribución de malformaciones congénitas, se determinó que el mayor porcentaje de estas fue $21 \%$ correspondiendo a 54 diferentes diagnósticos de malformaciones congénitas entre los que se puede mencionar hidranencefalia en 6 pacientes, microcefalia en 9 pacientes y gastrosquisis en 6 pacientes, entre otros diagnósticos, seguido de $16 \%$ correspondiente a 40 diagnósticos de hidrocefalia, $14 \%$ correspondiente a 36 pacientes con diagnóstico de mielomeningocele, $12 \%$ catalogados como síndrome dismorfogenético correspondiente a 30 pacientes, $11 \%$ correspondiente a 28 pacientes con diagnóstico de cardiopatía congénita, $8 \%$ correspondiente a 20 pacientes con diagnóstico de pie equino varo, $7 \%$ correspondiente a 19 pacientes con diagnóstico de labio hendido y $6 \%$ correspondiente a 17 pacientes con diagnóstico de síndrome de Down, de estos la totalidad de la población en estudio, 230 pacientes, afirmaron no poseer ninguna antecedente de malformaciones congénitas, no haber sido expuestas a rayos $\mathrm{x}$ y no tener ninguna enfermedad de transmisión sexual.

\section{Agradecimientos}

La investigación fue realizada con fondos propios de la investigadora. Se agradece de manera atenta al Hospital Nacional de Chiquimula especialmente al departamento de estadística la ayuda brindada para la elaboración y culminación de la presente investigación. De igual forma a los asesores del Centro Universitario de Oriente CUNORI. 


\section{Referencias bibliográficas}

Cisneros, G. \& Bosch, A. (2014). Alcohol, tabaco y malformaciones congénitas labioalveolopalatinas (en línea). MEDISAN 18(9):1293-1297. Consultado 29 mar. 2018. Disponible en http://scielo.sld.cu/ $\mathrm{pdf} / \mathrm{san} / \mathrm{v} 18 \mathrm{n} 9 / \mathrm{san} 15189 . \mathrm{pdf}$

Organización Mundial de la Salud, Suiza-OMS. (2015). Anomalías congénitas Ginebra, Suiza. Consultado 6 mar. 2018. Disponible http://www.who.int/es/news-room/fact-sheets/detail/congenital-anomalies.

Ortiz, M., Flores, G., Cardiel, L. \& Luna, C. (2003). Frecuencia de malformaciones congénitas en el área de neonatología del Hospital General de México. Revista Mexicana de Pediatría 70(3):128-131. Consultado 6 mar. 2018. Disponible en http://www.medigraphic.com/pdfs/pediat/sp-2003/sp033e. pdf.

Vega, A. \& Vizzuett, R. (2005). Frecuencia de malformaciones congénitas en el hospital general de tercer nivel. Revista Mexicana de Pediatría 72(2):70-73. Consultado 6 mar. 2018. Disponible en http:// www.medigraphic.com/pdfs/pediat/sp-2005/sp052d.pdf

\section{Sobre la autora}

\section{Glida Roosemary Aguilar Aroche}

Médico y Cirujano, egresada del Centro Universitario de Oriente CUNORI de la Universidad de San Carlos de Guatemala en el año 2018. Realizó la investigación que lleva como título "Caracterización de las pacientes con hijos diagnosticados con malformaciones congénitas. Actualmente se desempeña como médico residente 1 en el departamento de Diagnóstico por imágenes en el Hospital Roosevelt, Guatemala.

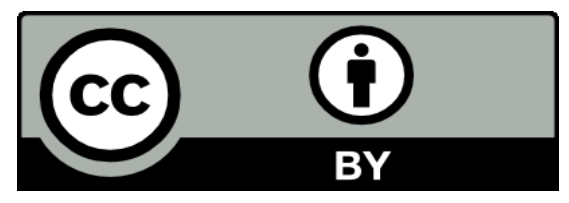

Este texto está protegido por una licencia CreativeCommons 4.0.

Usted es libre para compartir, copiar y redistribuir el material en cualquier medio o formato y adaptar el documento, remezclar, transformar y crear a partir del material para cualquier propósito, incluso comercialmente, siempre que cumpla la condición de atribución: usted debe reconocer el crédito de una obra de manera adecuada, proporcionar un enlace a la licencia, e indicar si se han realizado cambios. Puede hacerlo en cualquier forma razonable, pero no de forma tal que sugiera que tiene el apoyo del licenciante o lo recibe por el uso que hace. 